\title{
Sonic Hedgehog Facilitates Dopamine Differentiation in the Presence of a Mesencephalic Glial Cell Line
}

\author{
Nobuki Matsuura, ${ }^{1}$ D. Chichung Lie, ${ }^{2}$ Minoru Hoshimaru, ${ }^{1}$ Minoru Asahi, ${ }^{1}$ Masato Hojo, ${ }^{1}$ Ryuji Ishizaki, ${ }^{1}$ \\ Nobuo Hashimoto, ${ }^{1}$ Sumihare Noji, ${ }^{3}$ Hideyo Ohuchi, ${ }^{3}$ Hidefumi Yoshioka, ${ }^{3}$ and Fred H. Gage ${ }^{2}$ \\ ${ }^{1}$ Department of Neurosurgery, Kyoto University Graduate School of Medicine, 606-8507 Kyoto, Japan, 2Laboratory of \\ Genetics, The Salk Institute for Biological Studies, La Jolla, California 92037, and ${ }^{3}$ Department of Biological Science and \\ Technology, Faculty of Engineering, University of Tokushima, 770-0042 Tokushima, Japan
}

\begin{abstract}
The aim of this study was to establish a cellular system to investigate the requirement for cell surface and diffusible molecules in the differentiation of fetal mesencephalic cells toward the dopamine lineage. Toward this end, we immortalized rat embryonic day 14 (E14) mesencephalon with a regulatable retroviral vector encoding v-myc. The stably transduced cells were pooled and designated as VME14 cells. VME14 cells proliferated rapidly, stopped proliferating, extended processes, and expressed GFAP after suppression of the v-myc expression with tetracycline, suggesting that VME14 cells differentiated into glial cells. Dissociated cells derived from the E11 rat mesencephalon gave rise to only a small number of tyrosine hydroxylase (TH)-positive neurons. However, when grown on a monolayer of the differentiated VME14 cells, a significantly
\end{abstract}

Dopaminergic neurons that lie in the ventral midbrain play a key role in voluntary movement, emotional behavior, and cognition. Loss of these neurons is associated with Parkinson's disease. In experimental animal studies, grafts containing dopaminergic neurons dissected from the fetal ventral mesencephalon reversed some motor impairments in grafted animals with experimental Parkinson's disease (Yurek and Sladek, 1990; Dunnett, 1991; Bjorklund and Lindvall, 2000). On the basis of these studies, fetal nigral transplantation is now being used as an experimental therapy in treating patients with Parkinson's disease (Olanow et al., 1996). Although this method of neural replacement shows considerable promise, the extent of functional recovery attained in animal and clinical studies is partial and variable. The partial nature of the recovery has, for the most part, been attributed to a paucity of dopaminergic neurons in grafts and the relatively poor survival rate of grafted cells (Sladek et al., 1986; Yurek and Sladek, 1990). Indeed, it has been estimated that, in humans, only $5-6 \%$ of the total number of grafted ventral mesencephalic cells

\footnotetext{
Received Sept. 28, 2000; revised April 3, 2001; accepted April 4, 2001.

This research was supported in part by Ministry of Education, Science, and Culture of Japan Grant-in-Aid 08671578, the Japan Epilepsy Research Foundation, and the Cell Science Research Foundation. F.H.G. is supported by National Institutes of Health Grant AG08514, the Lookout Fund, and the National Parkinson Foundation. D.C.L. is supported by the Deutsche Forschungsgemeinschaft. We thank M. Gage for helpful critique of this manuscript and Linda Kitabayashi and Steve Forbes for excellent technical assistance. The SHH-blocking antibody was obtained from the Developmental Studies Hybridoma Bank (University of Iowa, Iowa City, IA).

N.M. and D.C.L. contributed equally to this work

Correspondence should be addressed to Dr. Fred H. Gage, The Salk Institute, Laboratory of Genetics, 10010 North Torrey Pines Road, La Jolla, CA 92037. E-mail: fgage@salk.edu.

Copyright (C) 2001 Society for Neuroscience $0270-6474 / 01 / 214326-10 \$ 15.00 / 0$
}

higher number of cells differentiated into TH-positive neurons. VME14 cells were transduced with the secreted N-terminal cleavage product of the Sonic hedgehog gene (SHH-N), an inducer of mesencephalic dopaminergic neurons. This monoclonal, SHH-N-overexpressing cell line further enhanced dopaminergic differentiation of E11 rat mesencephalon cells. Thus, $\mathrm{SHH}-\mathrm{N}$ and signals derived from fetal mesencephalic glia act cooperatively to facilitate dopaminergic differentiation. These fetal mesencephalon-derived cell lines will provide tools for the study of signals involved in dopaminergic differentiation.

Key words: differentiation; dopaminergic neuron; immortalization; mesencephalon; Parkinson's disease; tyrosine hydroxylase both survive and express the desired dopaminergic phenotype (Kordower et al., 1996). A more complete understanding of the extrinsic and intrinsic signals involved in dopaminergic cell fate determination will provide new strategies for increasing the number of dopaminergic neurons in mesencephalic grafts and/or improving the survival of engrafted fetal nerve cells.

Cells in the fetal ventral mesencephalon express neurotrophic factors for dopaminergic neurons, such as transforming growth factor- $\beta$ and glial cell line-derived neurotrophic factor (Poulsen et al., 1994). In addition, induction of mesencephalic dopaminergic neurons is mediated by the concerted action of diff usible and contact-dependent signals from the ventral mesencephalon (Hynes et al., 1995a). These findings have suggested that nonneural cells in the fetal ventral mesencephalon play an important role in generating and maintaining dopaminergic neurons in vivo and have prompted their use to increase the number of dopaminergic neurons in mesencephalic grafts. Although the availability of fetal mesencephalic tissues is limited, immortalization enables the generation of an unlimited supply of fetal mesencephalic cells. For immortalization, we used a retroviral vector LINXv-myc, in which the v-myc transgene is transcribed in a tetracyclineregulated manner (Hoshimaru et al., 1996). Neuronal progenitor cells immortalized by this retrovirus differentiate into neurons after the treatment with tetracycline (Hoshimaru et al., 1996). The present cells cultured from the rat ventral mesencephalon were immortalized by this retrovirus, and the efficacy of these immortalized cells in inducing tyrosine hydroxylase (TH)positive neurons was examined in primary dissociated cell culture of the fetal mesencephalon.

The N-terminal product of Sonic hedgehog autoproteolysis 
(SHH-N), which is secreted by the floor plate of the mesencephalon, induces dopaminergic neurons in the fetal mesencephalic explants in vitro (Hynes et al., 1995b; Wang et al., 1995). In addition, SHH-N promotes the survival of mesencephalic dopaminergic neurons and protects these cells from toxic insult (Miao et al., 1997). These findings have prompted us to test the effects of SHH-N overexpression in a feeder layer of immortalized cells from the mesencephalon.

\section{MATERIALS AND METHODS}

Production of retroviral producer cell line. The retroviral vector LXSHD/ $s h$-N was constructed by inserting chicken SHH-N cDNA (Kinto et al., $1997)$ into the polylinker site (XhoI and BamHI) of the retroviral vector LXSHD [generously provided by Dr. A. D. Miller (Fred Hutchinson Cancer Research Center, Seattle, WA)]. The selectable marker of LXSHD is histidinol dehydrogenase (Stockschlaeder et al., 1991).

All packaging and producer cell lines were cultured in DMEM with $10 \%$ fetal bovine serum at $37^{\circ} \mathrm{C}$ in a $10 \% \mathrm{CO}_{2}$ incubator. $\psi 2$ cells were transfected with $10 \mu \mathrm{g}$ of LXSHD/shh-N plasmid DNA using Trans IT polyamine transfection reagents (PanVera Corporation, Madison, WI). Virus-containing medium collected from $\psi 2$ cells $2 \mathrm{~d}$ after transfection was used to infect the amphotropic PA317 cells. One day after the infection, the cells were split at a 1:10 ratio, plated, and selected for L-histidinol (4 mM) resistance. Colonies were picked after selection for $8 \mathrm{~d}$ and were tested for the expression of the SHH-N transgene and the titer of the retrovirus.

Cell culture. All animal procedures were performed in accordance with guidelines of the Committee on Animal Experimentation of Kyoto University Graduate School of Medicine. Timed-pregnant rats were deeply anesthetized with an intraperitoneal injection of overdose pentobarbital, and fetuses were quickly collected. Pregnant rats were killed by exsanguination under deep anesthesia. The ventral mesencephalons of embryonic day 14 (E14) Wistar rats were dissected under a microscope. They were suspended in Dulbecco's PBS (Irvine Scientific, Santa Ana, CA) containing $0.2 \%$ trypsin (Sigma, St. Louis, MO), $0.2 \%$ dispase (Boehringer Mannheim, Mannheim, Germany), 0.2\% collagenase (Sigma), and $0.05 \%$ DNase I (Worthington, Freehold, NJ) and incubated in a $37^{\circ} \mathrm{C}$ water bath for $30 \mathrm{~min}$ with occasional shaking. After that, an equal volume of DMEM containing $10 \%$ fetal bovine serum and $0.1 \%$ trypsin inhibitor was added, and tissues were dissociated by repeated trituration with fire-polished Pasteur pipettes. Cells were then washed three times with DMEM-Ham's F-12 (Sigma) containing 0.05\% trypsin inhibitor. Then, cells were centrifuged at $1000 \times g$ for $5 \mathrm{~min}$, resuspended in DMEM-Ham's F-12 (1:1, v/v) medium containing 2.5 mM glutamine, N2 supplement (insulin at $5 \mu \mathrm{g} / \mathrm{ml}$, human transferrin at $50 \mu \mathrm{g} / \mathrm{ml}, 20 \mathrm{nM}$ progesterone, $100 \mu \mathrm{M}$ putrescine, and $30 \mathrm{~nm}$ sodium selenite) (Life Technologies, Gaithersburg, MD), and $20 \mathrm{ng} / \mathrm{ml}$ basic fibroblast growth factor (bFGF) (human recombinant; R \& D Systems, Minneapolis, MN), and plated on plastic tissue culture dishes coated with poly-D-lysine and laminin (Becton Dickinson, Mountain View, CA). Cells were incubated at $37^{\circ} \mathrm{C}$ in a $5 \% \mathrm{CO}_{2}$ incubator. Primary hippocampal glial cells were isolated as described previously (Goslin et al., 1998).

Retroviral-mediated gene transfer into ventral midbrain cells. Mesencephalic cells from E14 rats were allowed to grow at $37^{\circ} \mathrm{C}$ for $3 \mathrm{hr}$ after plating and were incubated with the conditioned medium of the LINXvmyc producer cells in the presence of $4 \mu \mathrm{g} / \mathrm{ml}$ polybrene for $6 \mathrm{hr}$ (Hoshimaru et al., 1996). After infection, the conditioned medium was replaced with N2 plus bFGF medium. Cells were incubated until cell culture became $70-80 \%$ confluent and were selected for G418 (100 $\mu \mathrm{g} / \mathrm{ml})$ resistance. Colonies were collected and analyzed immunohistochemically.

A pooled population of mesencephalic cells transduced with the LINXv-myc retroviral vector was subsequently incubated with the $\mathrm{LXSHD} / s h h-\mathrm{N}$ retrovirus in the presence of $4 \mu \mathrm{g} / \mathrm{ml}$ polybrene for $18 \mathrm{hr}$. One day after infection, the cells were split at a 1:10 ratio, plated, and selected for L-histidinol (4 mM) resistance. Colonies were picked after selection for $7 \mathrm{~d}$ and analyzed.

Northern blot analysis. Cells were homogenized in $5 \mathrm{ml}$ of the total RNA isolation reagent (TRIzol Reagent; Life Technologies) per $100 \mathrm{~mm}$ dish. Total RNA $(20 \mu \mathrm{g})$ was separated on $1 \%$ agarose-formaldehyde gels and transferred onto a nylon membrane (Pall Biodyne transfer membrane; Pall Gelman Laboratory Inc., Ann Arbor, MI ). Hybridization and subsequent washes were performed as described previously (Kojima et al., 1996). The membranes were probed with chicken SHH-N cDNA (Kinto et al., 1997).

Immunocytochemistry. Paraformaldehyde-fixed cells were blocked overnight at $4{ }^{\circ} \mathrm{C}$ in TBS containing $0.3 \%$ Triton X-100 and $5 \%$ preimmune donkey serum or $5 \%$ goat serum depending on the secondary antibody. Cells were incubated with primary antibodies for $24 \mathrm{hr}$ at $4{ }^{\circ} \mathrm{C}$, washed five times with TBS, and incubated with secondary antibodies conjugated to fluorescein isothiocyanate, rhodamine, Texas Red, or cyanin 5 for $24 \mathrm{hr}$. Samples were washed five times and treated with 10 $\mathrm{mg} / \mathrm{ml} \mathrm{4}$ ', 6-diamidino-2-phenylindole (DAPI) (Sigma). Bromodeoxyuridine (BrdU) visualization was performed as described previously (Takahashi et al., 1999).

Primary antibodies used were mouse anti-type III $\beta$-tubulin (TuJ1) (1:1000; Babco, Richmond, CA), mouse anti-nestin $(0.2 \mu \mathrm{g} / \mathrm{ml}$; PharMingen, San Diego, CA), mouse anti-neurofilament $200 \mathrm{kDa}(\mathrm{NF}-\mathrm{H})(2.0$ $\mu \mathrm{g} / \mathrm{ml}$; Boehringer Mannheim), mouse anti-TH (1:200; Chemicon, Temecula, CA), rabbit anti-TH (1:500; Protos Biotech, New York, NY), rat anti-BrdU (1:200; Accurate Chemicals, Westbury, NY), rabbit anti-glial fibrillary acidic protein (GFAP) (1:1; Zymed, San Francisco, CA), guinea pig anti-GFAP (1:500; Advanced Immunochemical Inc., Long Beach, CA), and rabbit anti-v-myc (1:8000; Upstate Biotechnology, Lake Placid, NY). Secondary antibodies were used at 1:200 (goat; Organon TeknikaCappel, Durham, NC) or 1:500 (donkey; Jackson ImmunoResearch, West Grove, PA) dilution.

Terminal deoxynucleotidyl transferase-mediated biotinylated UTP nick end labeling staining. DNA fragmentation was detected in situ using the immunocytochemical terminal deoxynucleotidyl transferase-mediated biotinylated UTP nick end labeling (TUNEL) technique (Boehringer Mannheim) according to the protocol of the manufacturer. As a negative control, some cells were processed in the buffered nucleotide mixture in the absence of terminal deoxynucleotidyl transferase enzyme.

Immunoblot analysis for SHH-N. SHH-N in conditioned medium was detected as described previously (Kinto et al., 1997). Briefly, immunoblotting was performed after enrichment of SHH-N with heparin-agarose. SHH-N was detected using affinity-purified antibody raised against the N-terminal residue of chicken SHH-N (103-170) (Kinto et al., 1997) and the ECL Western blotting system (Amersham Pharmacia Biotech, Buckinghamshire, UK).

Reverse transcription-PCR analysis. One microgram of total RNA was subjected to reverse transcription (RT)-PCR analysis. RT reaction and PCR were performed using the RT-PCR kit (TaKaRa, Kyoto, Japan). The RT-PCR products were separated on $1.8 \%$ agarose gels and stained with ethidium bromide. Control experiments using primers for the rat ribosomal protein L27a (internal control) showed that the amount of amplified RT-PCR product was directly proportional to the amount of input RNA within the range of $0.5-4 \mu \mathrm{g}$ after 28 cycles of amplification (data not shown). The sequences of genomic DNA between two primers were selected so that they contained one or two introns for discrimination between products from RNA and contaminating genomic DNA. The following oligonucleotides were used as primers: rat ribosomal protein L27a 5' primer, 5'-ATCGGTAAGCACCGCAAGCA-3' and 3' primer, 5'-GGGAGCAACTCCATTCTTGT-3'; rat GFAP 5' primer, 5'-AC CTCGGCACCCTGAGGCAG-3' and $3^{\prime}$ primer, 5'-CCAGCGACTCAACCTTCCTC-3'; rat NF-H 5' primer, 5'-GAGGAGATAACT GAGTACCG-3' and 3' primer, 5'-CCAAAGCCAATCCGACACTC-3' (Hoshimaru et al., 1996); rat TH 5' primer, 5'-CTGGAGGCTGTGG TCTTTGAGGAG-3' and $3^{\prime}$ primer, 5'-CAGTACCGTTCCAGAAGC TG-3'; rat SHH 5'primer, 5'-GGAAGATCACAAGAAACTCC GAAC-3' and 3' primer, 5'-GGATGCGAGCTTTGGATTCATAG-3'; rat FGF8 5' primer, 5'-CATGTGAGGGACCAGAGCC-3' and 3' primer, 5'-GTAGTTGTTCTCCAGCAGGATC-3' (Studer et al., 2000); and rat GDNF 5' primer, 5'-ATGGGATGTCGTGGCTGTCTG-3' and 3' primer, 5'-TCCTCCTTGGTTTCGTAGCCC-3'. Specificity of reactions was verified by directly sequencing PCR products for L27a, GFAP, NF-H, and TH using PCR cycle sequencing followed by electrophoresis on an Applied Biosystems (Foster City, CA) Prism 377 DNA sequencer.

Coculture of embryonic mesencephalic cells with immortalized cells. VME14 or A1 cells (see below) were plated at $0.5 \times 10^{-5}$ cells per well onto eight-well Lab-Tek glass chamber slides coated with poly-D-lysinelaminin in the presence of tetracycline $(1 \mu \mathrm{g} / \mathrm{ml})$. After $1 \mathrm{~d}$, these cells formed a monolayer. At this time, the E11 rat mesencephalons were dissociated as described above and plated onto the monolayer at $0.25 \times$ $10^{-5}$ or $0.5 \times 10^{-5}$ cells per well. Control cultures were plated alone onto glass slides at $0.75 \times 10^{-5}$ or $10^{5}$ cells per well. To study the effect 
of different plating densities, cells were plated onto the monolayer at densities ranging from $10^{3}$ to $10^{5}$ cells per well.

Blocking experiments were performed using SHH-N blocking antibodies (Developmental Studies Hybridoma Bank, University of Iowa, Iowa City, IA) at $20 \mu \mathrm{g} / \mathrm{ml}$ (Ericson et al., 1996). To exclude unspecific effects of antibodies, control cultures were treated with $10 \mu \mathrm{g} / \mathrm{ml}$ donkey antimouse IgG (Jackson ImmunoResearch).

Dividing cells were marked by adding BrdU (10 $\mu$ m; Sigma) on day 1 in vitro (DIV). Immunofluorescence studies were performed after 2 and 4 DIV.

Quantification and statistical analysis. The number of TH-positive cells generated in each condition was quantified in two ways. At a plating density of $0.5 \times 10^{-5}$ cells per well, the number of cell clusters that contained TH-positive cells was counted in each culture of cells derived from E11 mesencephalon. At a plating density of $0.25 \times 10^{-5}$ cells per well, which allowed counting of individual cells, the absolute number \pm SEM of TH-positive cells or TUNEL-positive cells per visual field was determined by counting six random fields at $20 \times$ magnification. The number of TUNEL-positive cells in the coculture conditions was adjusted for plating density of E11 cells and contribution of A1/VME14 cells to the total number of TUNEL-positive cells. Colocalization of TUNEL signal with $\mathrm{TH}$ expression or TuJ1 expression was evaluated by confocal analysis of the entire culture. The percentage of TH-TUNEL or TuJ1-TUNEL double-labeled cells per TH-positive cells or TuJ1positive cells was calculated for each condition. BrdU incorporation by TH-positive neurons was evaluated by confocal analysis. The percentage of TH-BrdU double-labeled cells per TH-positive cells was calculated for each condition. The data were evaluated statistically by ANOVA and followed by Student's $t$ test using the Stat-View 4.5 software (Abacus Concepts, Calabasas, CA). Differences were considered significant when $p$ values were $<0.05$.

Quantification of TH-positive cells in individual clusters was performed by imaging with a Bio-Rad (Hercules, CA) MRC1024UV confocal imaging system. Z-series were taken at $8 \mu \mathrm{m}$ intervals, and pictures were converted to an Adobe Photoshop file (Adobe Systems, San Jose, CA). TH-positive cells and DAPI-positive nuclei were counted, and the percentage of TH-positive cells per DAPI-positive nuclei was calculated.

\section{RESULTS}

\section{Conditional immortalization of rat mesencephalic cells}

Cells cultured from the ventral mesencephalon of E14 rats were infected with the LINXv-myc retrovirus. Colonies that appeared after selection for G418 were pooled and designated as VME14. VME14 cells were rounded and polygonal in shape and did not have processes (Fig. 1A). VME14 cells grew rapidly (doubling time of $12 \mathrm{hr}$ ). However, $1 \mathrm{~d}$ after the addition of tetracycline, most of the cells stopped dividing and began extending processes. After $5 \mathrm{~d}$, processes became more noticeable (Fig. $1 B$ ). To test whether these changes in morphology were attributable to the differentiation induced by downregulation of the v-myc transgene, the cells were examined immunohistochemically. When the cells were grown in the absence of tetracycline, immunoreactivity of $\mathrm{v}-m y c$ was observed in the nuclei of almost all of the cells, although the cells were not uniformly stained because of the polyclonal origin of the cells (Fig. 1C). When the cells were grown in the presence of tetracycline, the v-myc immunoreactivity was localized to the cytoplasm and became faint, indicating that the LINXv-myc retroviral vector functioned properly (Fig. 1D). Strong immunoreactivity of nestin was exhibited in the cytoplasm of almost all of the cells, indicating that these cells were neural precursor cells (Fig. 1E). The anti-nestin antibody stained filamentous structures in the cells grown in the presence of tetracycline (Fig. $1 F$ ). On the other hand, only a few cells in culture were cytoplasmically stained by the anti-GFAP antibody when grown in the absence of tetracycline (Fig. 1G). When the cells were grown in the presence of tetracycline, this antibody stained many cells bearing prominent processes, indicating that VME14 cells differentiated into glial cells (Fig. $1 H$ ). In parallel to the immu- nological studies, RT-PCR analyses showed the induction of GFAP mRNA in the VME14 cells after the addition of tetracycline (Fig. 2). Immunoreactivity of NF-H was observed in a few cells that did not show neuronal morphology in culture, regardless of the tetracycline treatment (Fig. 1I,J). RT-PCR analyses also showed the expression of NF-H mRNA in VME14 cells grown in either the absence or presence of tetracycline (data not shown). No TH-positive cells were observed in cultures, regardless of the tetracycline treatment, and RT-PCR analysis also failed to demonstrate TH expression of mRNA (data not shown).

\section{Isolation of immortalized mesencephalic cells expressing $\mathrm{SHH}-\mathrm{N}$ transgene}

An amphotropic producer cell line producing LXSHD/shh-N retrovirus was selected and expanded. The virus had a titer of $10^{6}$ colony-forming units per milliliter for NIH3T3 cells and did not contain helper viruses. VME14 cells were transduced with this retrovirus and selected for stable transfectants with L-histidinol (4 $\mathrm{mm}$ ). Colonies were picked after selection for $7 \mathrm{~d}$ and tested for the expression of SHH-N mRNA, which was transcribed from the retroviral long terminal repeat (4.0 kb in length). A clone, A1, showed high mRNA expression of the SHH-N transgene (Fig. $3 B$ ). On the other hand, endogenous expression of Sonic hedgehog mRNA could not be detected in VME14 cells by RT-PCR using rat SHH-specific primers (Studer et al., 2000) (Fig. 3A) or Northern blot analysis (Fig. $3 B$ ). Immunoblot analysis for the medium conditioned by A1 cells demonstrated that the SHH-N protein was secreted by A1 cells (Fig. 3C). In contrast, VME14 cells did not secrete a detectable amount of the SHH-N protein (Fig. $3 C$ ). A1 cells stopped dividing and extended processes $1 \mathrm{~d}$ after the addition of tetracycline as VME14 cells did. GFAP immunoreactivity was not observed in A1 cells grown in the absence of tetracycline and was exhibited in some A1 cells after treatment with tetracycline (data not shown). RT-PCR analyses also demonstrated the induction of GFAP mRNA after treatment with tetracycline (Fig. 2). A few NF-H-positive cells and no $\mathrm{TH}$-positive cells were observed in culture of A1 cells grown in either the absence or presence of tetracycline (data not shown).

\section{Increase in TH-positive cell numbers by coculture with A1 and VME14 cells}

VME14 or A1 cells showed contact inhibition $1 \mathrm{~d}$ after plating in the presence of tetracycline and maintained a monolayer, which was used as a feeder layer. After $5 \mathrm{~d}$, no TH immunoreactivity was seen in this monolayer (data not shown).

Dissociated cell cultures derived from the whole mesencephalon of E11 rats developed cell clusters after 2 DIV. Cell clusters increased in size after 4 DIV and contained many cells that stained for the early neuronal marker TuJ1 (Figs. 4, 5A). Occasionally, TH-positive cells were observed but were faintly stained, and the number never exceeded two TH-positive cells per cluster (Fig. 5A).

When dissociated cells from E11 mesencephalon were grown on a monolayer of VME14, cell clusters were more prominent after 2 DIV. After 4 DIV, cell clusters increased in size and developed more prominent processes compared with dissociated cells grown alone (Fig. 4). Most strikingly, the number of THpositive cells greatly increased, as reflected by the increased total number of TH-positive cell clusters and the appearance of cell clusters that contained more than two TH-positive cells per cluster (19.6 $\pm 3.8 \%$ SEM of total number of cell clusters). This pronounced effect on the generation of $\mathrm{TH}$-positive cells from 

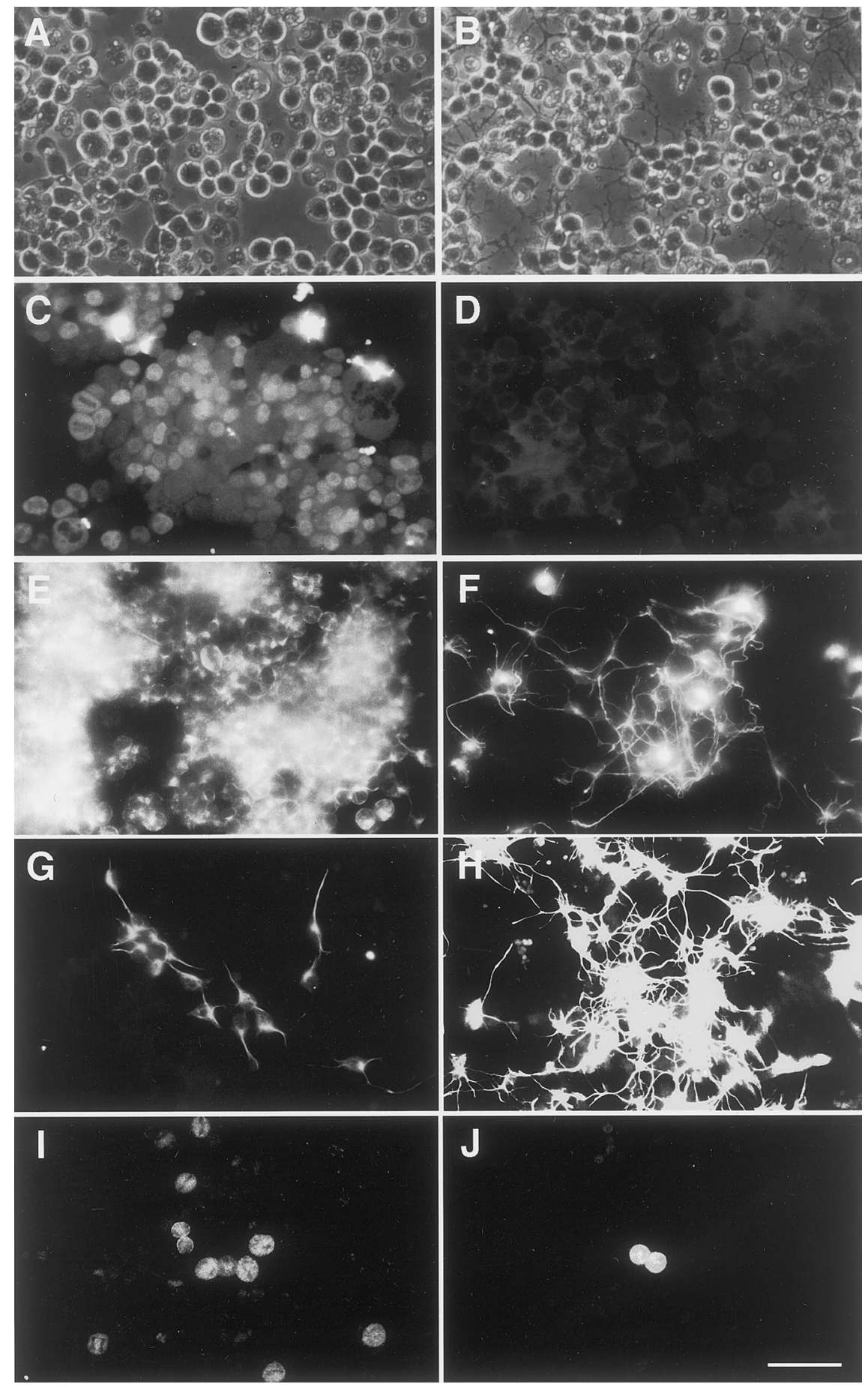

Figure 1. Microphotographs showing E14 ventral mesencephalic cells transduced with LINXv-myc retrovirus (VME14 cells) grown in the absence of tetracycline for $2 \mathrm{~d}(A, C, E, G, I)$ or in the presence of tetracycline $(1 \mu \mathrm{g} / \mathrm{ml})$ for $5 \mathrm{~d}(B, D, F, H, J)$. The cells were stained with anti-v-myc antibody $(C, D)$, antinestin antibody $(E, F)$, anti-GFAP antibody $(G, H)$, and anti-NF-H antibody $(I, J)$. Scale bar, $50 \mu \mathrm{m}$.
E11 mesencephalic cells was significantly augmented by cocultures with SHH-N-overexpressing VME14 (A1) cells (35.3 \pm $0.7 \%$ of total number of cell clusters; $p<0.005$ ) (Figs. $5 B, 6$ ). Under these conditions, clusters contained up to $>1000$ cells, of which as much as $5.2 \%$ of the cells were TH-positive. Primary hippocampal glia derived from postnatal day 0 rat pups were also able to stimulate $\mathrm{TH}$ expression in E11 mesencephalic cells but were far less effective in the generation of $\mathrm{TH}$-positive clusters containing more than two TH-positive cells $(10.5 \pm 2.7 \%$ of total number of cell clusters) compared with cocultures with A1 cells $(p<0.001)$ or VME14 cells $(p<0.05)$ (Fig. 6), indicating that A1 or VME14 produces a specific activity that acts on mesence- phalic precursors. Conditioned media from differentiated VME14 and A1 cells failed to reproduce the effects of the feeder layer (data not shown).

Initial experiments had shown that $\mathrm{TH}$-positive neurons could be observed in cocultures over a broad range of plating densities $\left(10^{3}\right.$ to $10^{5}$ E11 cells per well) and that higher plating densities of E11 cells were accompanied by a significant increase in the number of TH-positive neurons. Moreover, A1 cocultures constantly yielded significantly more TH-positive neurons than VME14 cocultures after 4 DIV, except at a plating density of $10^{3}$ E11 cells per well, which was most likely attributable to the overall low numbers of TH-positive neurons in this condition 


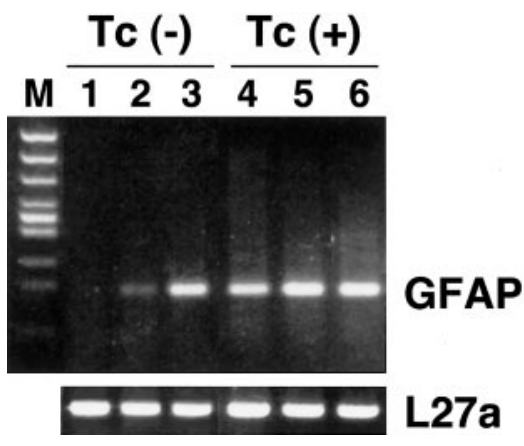

Figure 2. RT-PCR analysis. Primers for GFAP exhibit PCR products of 141 bp in A1 cells $(1,4)$, VME14 cells $(2,5)$, and the adult rat forebrain $(3,6)$. The cells were grown in the absence $[T c(-)]$ or presence $[T c(+)]$ of tetracycline $(1 \mu \mathrm{g} / \mathrm{ml})$. Primers for L27a were used as an internal control. $M$, Bacteriophage $\varnothing 174$ DNA HincII digests.

(data not shown). Given that the effect of the cocultures was independent of the plating density, E11 cells were plated at lower densities $\left(0.25 \times 10^{-5}\right.$ cells per well in coculture conditions; $0.75 \times 10^{-5}$ cells per well in control conditions) to allow a more accurate quantification of TH-positive cells. Under these conditions, cocultures with VME14 cells generated an approximately twofold increase in the number of TH-positive cells (39.2 \pm 5.8 TH-positive cells per visual field) compared with E11 cells grown alone $(19.5 \pm 1.9 \mathrm{TH}$-positive cells per visual field $)$. A1 cocultures increased this effect by $\sim 50 \%(62.0 \pm 2.5 \mathrm{TH}$-positive cells per visual field) (Figs. 7, 8). Given the threefold difference in the number of plated E11 cells $\left(0.25 \times 10^{-5}\right.$ cells per well in coculture conditions vs $0.75 \times 10^{-5}$ cells per well in control conditions), these numbers underestimate the efficiency of the coculture conditions considerably.

\section{Mechanisms involved in increased TH expression conferred by VME14 cells and A1 cells}

To determine the mechanism by which cocultures with VME14 and A1 cells increased the number of TH-positive cells from E11 midbrain cells, we first compared the effects of the cocultures after 2 and 4 DIV. When cultures were analyzed after 2 DIV, a significantly higher number of TH-positive cells was observed in A1 cocultures compared with VME14 cocultures $(p<0.05)$ and E11 cells grown alone $(p<0.005)$. VME14 cocultures also produced a higher average of TH-positive neurons after 2 DIV compared with control conditions; however, at this time point, the difference between these two conditions was not statistically significant ( $p=0.102)$ (Fig. 8). After 4 DIV, the number of TH-positive neurons increased significantly in each culturing condition (Fig. 8). To determine whether coculture conditions favored the proliferation of dopaminergic precursors, cultures were treated with BrdU continuously from day 1 in vitro. After 2 and 4 DIV, $<1 \%$ of the TH-positive neurons had incorporated BrdU in any of the culture conditions, indicating that the vast majority of the TH-positive neurons was not derived from a proliferating population (Fig. 7). The number of TUNEL-positive cells did not differ significantly after 2 and 4 DIV between E11 cells in VME14 cocultures and E11 cells grown alone (Fig. 9). This finding indicates that the increased yield of $\mathrm{TH}$ neurons from VME14 cocultures is not primarily caused by enhanced survival of TH-positive neurons or dopaminergic precursors. In A1 cocultures, the number of TUNEL-positive cells was significantly lower after 4 DIV compared with other culture

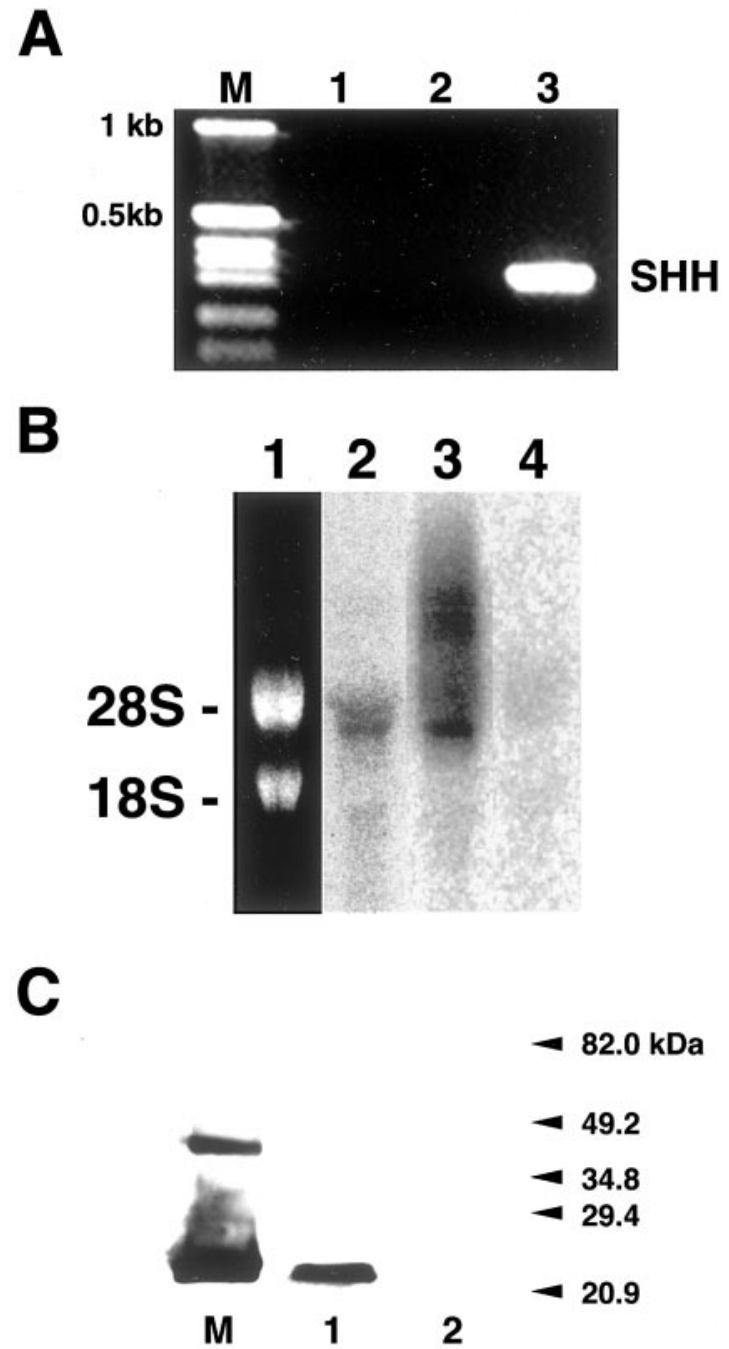

Figure 3. A, RT-PCR for SHH mRNA using rat SHH-specific primers. No rat SHH mRNA is detected in VME14 cells (1) or A1 cells (2). Marker $(M)$ and positive control, E12 rat brain (3). B, Northern blot analysis using total RNA exhibits the expression of SHH-N mRNA transcribed from the long terminal repeat of the LXSHD/SHH-N retrovirus (4.0 kb in length) in the LXSHD/SHH-N producer cells (2) and A1 cells (3). The slower migrating band corresponds to a larger transcript from the viral long terminal repeat, which contains the SHH-N transgene and the histidinol resistance gene. No expression of endogenous Sonic hedgehog mRNA is demonstrated in VME14 cells (4). A gel stained with ethidium bromide is also presented (1). C, Secretion of SHH-N protein. Western blot analysis using an anti-SHH-N antibody demonstrates SHH-N protein $(23 \mathrm{kDa})$ in medium conditioned by A1 cells (1) but not in medium conditioned by VME14 cells (2). Purified recombinant mouse SHH-N is presented as a positive control $(M)$.

conditions (Fig. 9), suggesting that SHH-N might support overall survival of cells at later stages. However, significantly more TUNEL-positive cells were observed in A1 cocultures after 2 DIV (Fig. 9). Moreover, no TUNEL signal was detected after 2 and 4 DIV in TH-positive neurons under either culture condition, and very few $(<0.1 \%)$ TuJ1-positive neurons were labeled by TUNEL staining. It is therefore unlikely that the increased number of TH-positive cells in A1 cocultures is primarily a function of increased survival of $\mathrm{TH}$ positive neurons mediated by SHH-N.

To exclude that the clonal A1 cell line represents a subpopulation of VME14 cells that has enhanced activity regarding the 

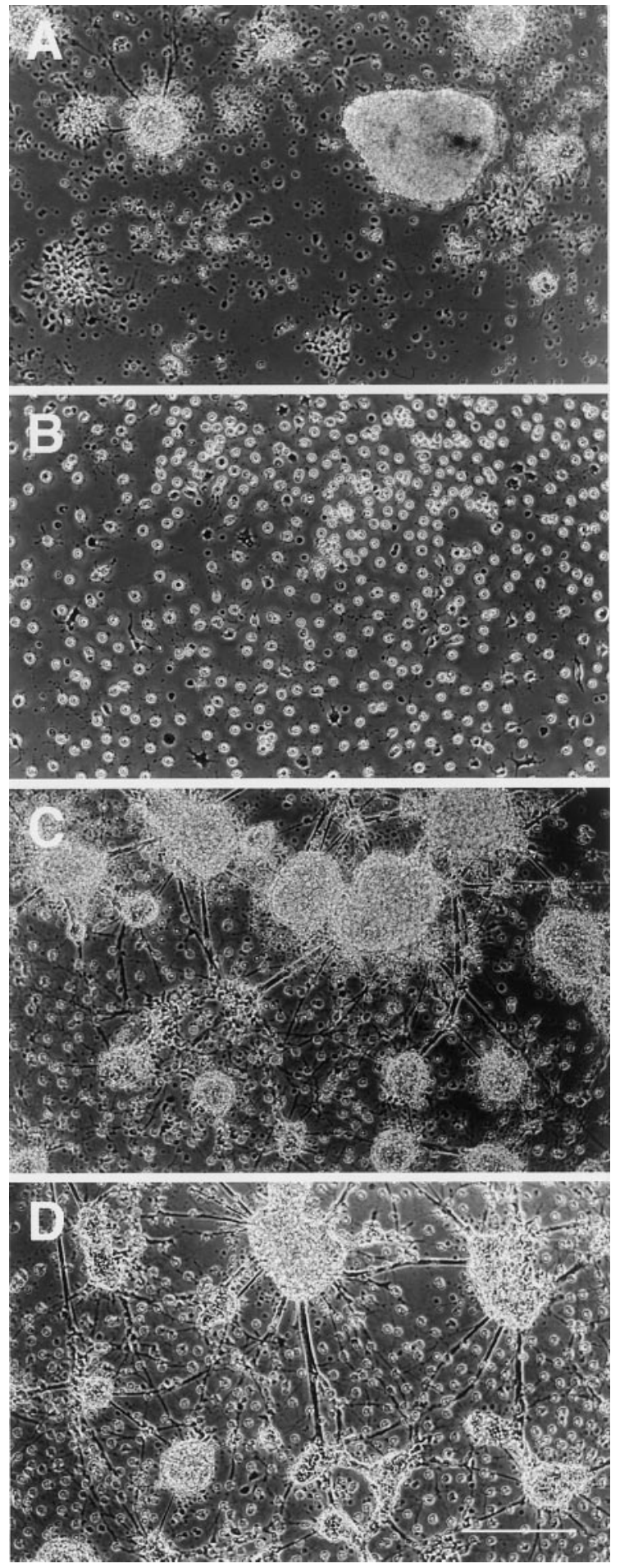

Figure 4. Microphotographs showing E11 mesencephalic cells grown alone and grown on a monolayer of VME14 and A1 cells. E11 mesencephalic cells extended a few neurites when grown alone for $4 \mathrm{~d}(A)$. A1 cells maintained a monolayer when grown in the presence of tetracycline for $4 \mathrm{~d}(B)$. Clusters of E11 mesencephalic cells were well developed and interconnected by neurites when grown on a monolayer of VME14 $(C)$ and $\mathrm{A} 1(D)$ cells for $4 \mathrm{~d}$. Scale bar, $250 \mu \mathrm{m}$.

generation of TH-positive neurons and to further demonstrate the additive actions of SHH-N, A1 cocultures were treated with high concentrations of SHH-N blocking antibodies. Treatment with specific blocking antibodies resulted in significantly lower
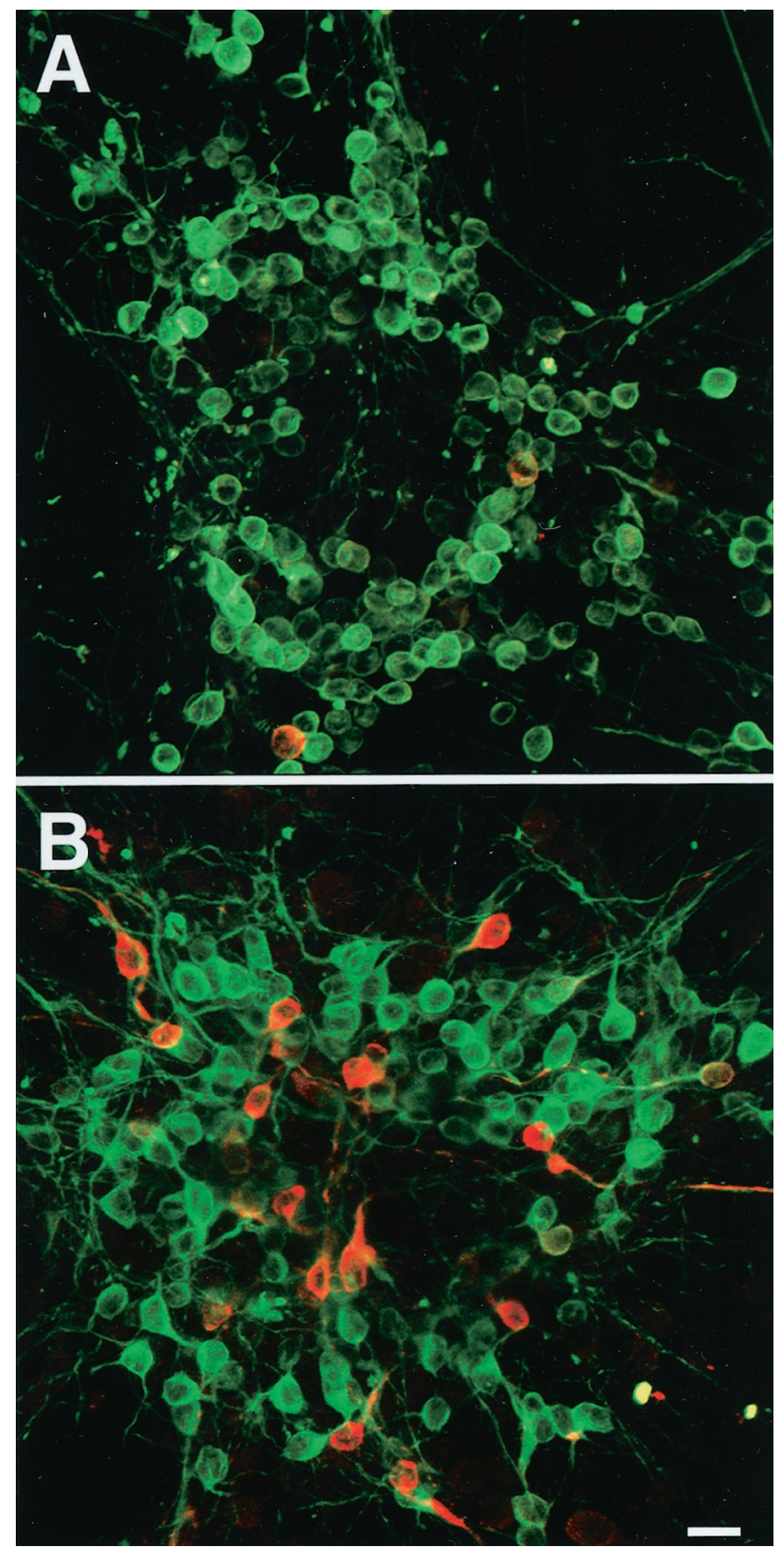

Figure 5. Immunofluorescence staining for TH (red) and TuJ1 (green). Few TH and TuJ1 double-labeled neurons were observed in E11 mesencephalic cells grown alone for $4 \mathrm{~d}(A)$. More TH-positive neurons were observed after $4 \mathrm{~d}$ in cultures of E11 mesencephalic cells grown on a monolayer of A1 cells $(B)$. Scale bar, $20 \mu \mathrm{m}$.

numbers of TH-positive neurons in A1 cocultures compared with nontreated A1 cocultures $(p=0.005)$ and unspecific antibodytreated A1 cocultures $(p<0.05)$. In addition, there was no statistical difference between specific antibody-treated A1 cocultures and VME14 cocultures $(p=0.2382)$. This effect cannot be attributed to toxicity of the SHH-N blocking antibody, because treatment of VME14 cocultures with this antibody did not result in a lower number of TH-positive neurons compared with untreated VME14 cocultures (Fig. 10). 


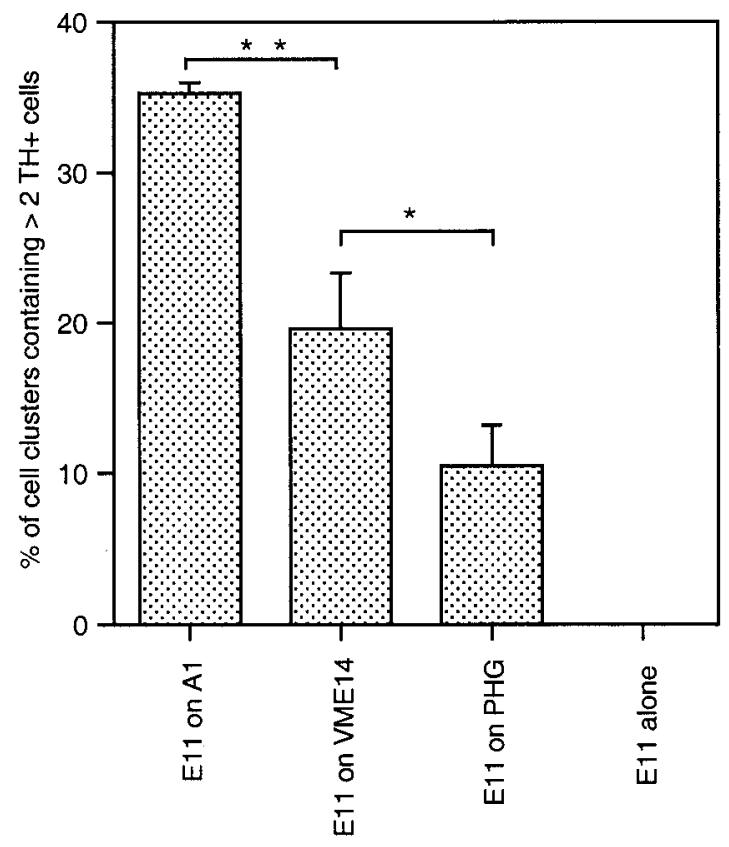

Figure 6. Histogram demonstrating the percentage of cell clusters containing more than two TH-positive $(\mathrm{TH}+)$ cells in cultures of E11 mesencephalic cells grown alone and grown on a monolayer of A1, VME14, or primary hippocampal glial $(P H G)$ cells. The percentage of clusters of E11 mesencephalic cells containing more than two TH-positive cells was significantly higher on a monolayer of VME14 cells than on that of PHG cells $\left({ }^{*} p<0.05\right)$. Cocultures with A1 cells increased the percentage of clusters containing more than two TH-positive cells significantly compared with cocultures with VME14 cells $(* * p<0.005)$.

\section{DISCUSSION}

\section{Dissociated cell cultures of E11 mesencephalon give rise to TH-positive neurons}

During development, rat ventral mesencephalic dopaminergic precursors begin to express $\mathrm{TH}$ at approximately E12.5 (Specht et al., 1981). Although there is evidence that dopaminergic precursors, identified by expression of aldehyde dehydrogenase 2, exist as early as E9.5 in ventral midbrain of mice (corresponding to approximately E10.5 in rats) (Wallen et al., 1999), attempts to generate dopaminergic neurons from time points earlier than E12 have not been successful (Murphy et al., 1990; Nakafuku and Nakamura, 1995). In the present study, we demonstrate that primary dissociated cells derived from rat E11 mesencephalon can give rise to a small number of TH-positive neurons. These results indicate that, in E11 rats, a small proportion of dopaminergic precursors is no longer dependent on extrinsic signals regarding dopaminergic differentiation and that our culturing conditions promoted the survival of dopaminergic precursors more efficiently than previously described cell culture systems.

\section{Ventral mesencephalic glia and SHH-N increase the yield of TH-positive neurons in vitro}

Several studies have underlined the importance of diffusible and contact-dependent signals from the ventral mesencephalon in the induction (Hynes et al., 1995a,b; Ye et al., 1998), differentiation (Wagner et al., 1999), and survival (O'Malley et al., 1992; Takeshima et al., 1994; Engele et al., 1996; Panchision et al., 1998; Wagner et al., 1999) of mesencephalic dopaminergic precursors. We therefore hypothesized that ventral mesencephalon can increase the number of E11 mesencephalic precursors differentiat-
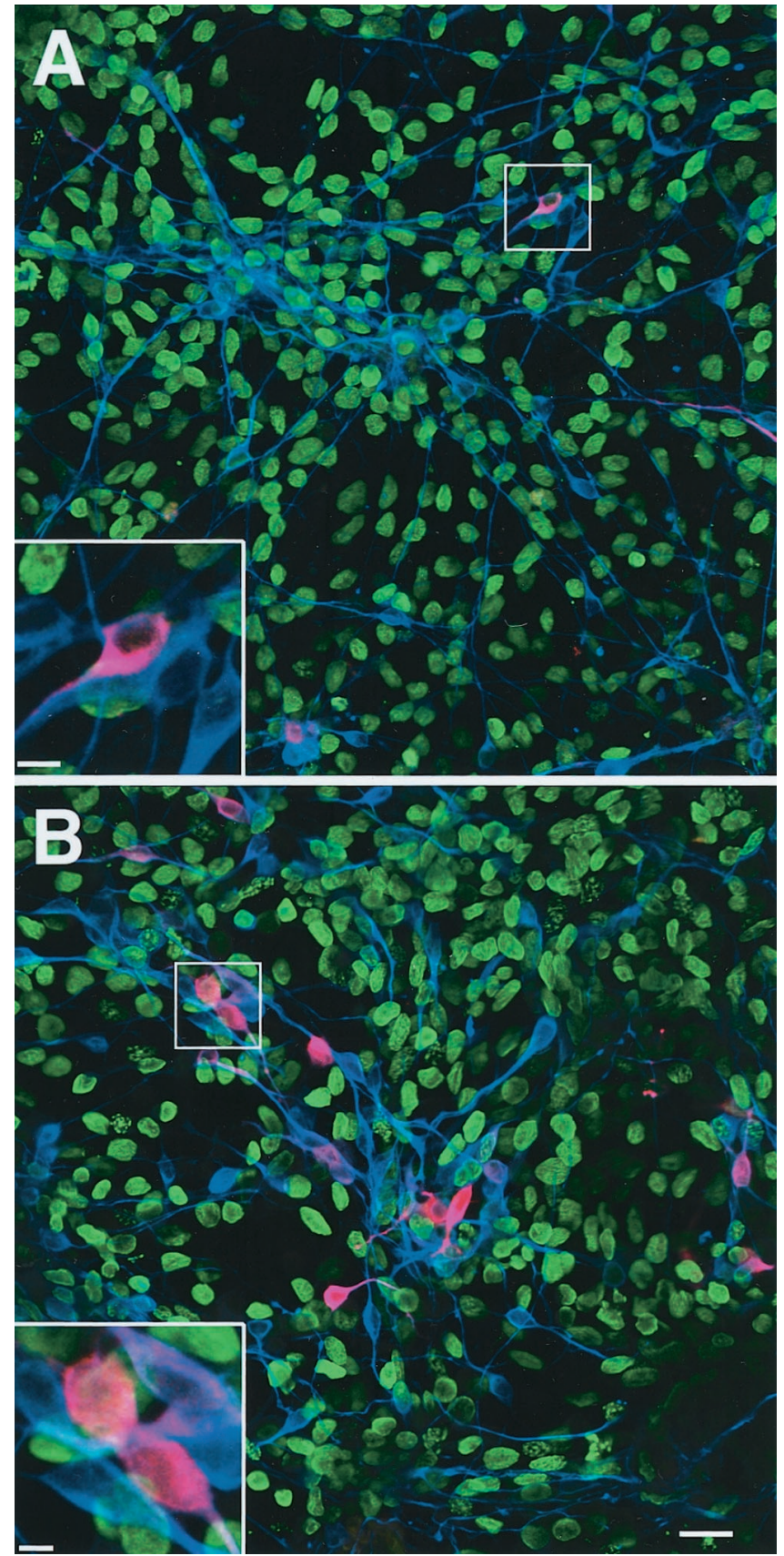

Figure 7. Immunoflourescence staining for BrdU ( green), $\mathrm{TH}$ (red), and TuJ1 (blue). Compared with E11 cells grown alone $(A)$, E11-A1 cocultures $(B)$ generated more TH-positive neurons (magenta) after 4 DIV. Higher magnification showed that TH-positive cells did not incorporate $\mathrm{BrdU}$, demonstrating that these cells were not derived from a proliferating population in vitro. Scale bars: $B, 20 \mu \mathrm{m}$; insets, $5 \mu \mathrm{m}$.

ing into dopaminergic neurons in vitro. In addition, we tested the additional effects of SHH-N in this paradigm, because SHH-N induces dopaminergic neurons in rat E9 midbrain explants (Hynes et al., 1995b) and supports survival of differentiated dopaminergic neurons (Miao et al., 1997).

To obtain an unlimited supply of fetal cells from the ventral mesencephalon, we took advantage of the LINXv-myc retrovirus, which has proven to be useful for conditional immortalization of neuronal progenitor cells (Hoshimaru et al., 1996). We successfully immortalized cells of the E14 ventral mesencephalon and 


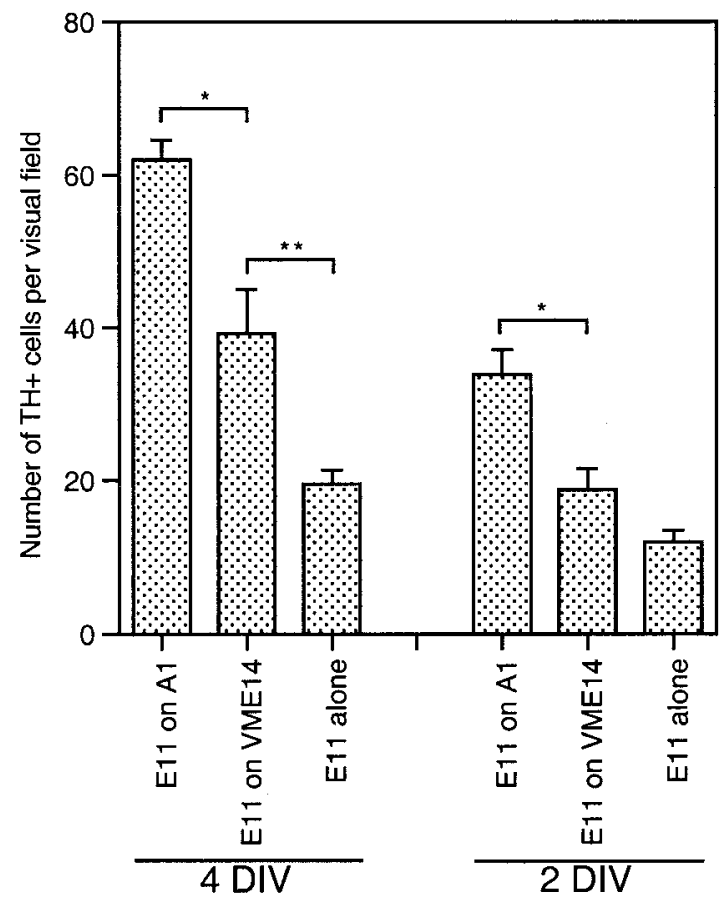

Figure 8. Histogram showing the number of TH-positive $(\mathrm{TH}+)$ cells per visual field at $20 \times$ magnification. The number of TH-positive cells increased in each condition between 2 and 4 DIV. Coculture with VME14 or A1 cells yielded a higher number of TH-positive cells than E11 cells grown alone, with A1 cells being more effective at each time point $\left({ }^{*} p<0.05 ;{ }^{* *} p<0.01\right)$.

obtained a population that could be differentiated into glial cells after treatment with tetracycline. Furthermore, a monoclonal cell line that overexpressed and secreted $\mathrm{SHH}-\mathrm{N}$ was generated from the VME14 cells.

As hypothesized, a feeder layer of VME14 was able to significantly increase the number of TH-positive neurons in dissociated cell cultures from E11 mesencephalon. This increase was even more pronounced when the feeder layer secreted SHH-N. Together, these results suggest that unidentified factors other than SHH-N are essential for this effect because VME14 cells, which did not secrete a detectable amount of SHH-N, also increased the yield of TH-positive neurons.

The increase in TH-positive cells by the feeder layer and SHH-N could reflect different phenomena: induction of dopaminergic precursors, increased differentiation of existing dopaminergic precursors, increased proliferation of dopaminergic precursors, and increased survival of dopaminergic precursors or TH-positive neurons. The present results, as well as previous findings, that showed that $\mathrm{SHH}-\mathrm{N}$ induced dopaminergic precursors in explant cultures (Hynes et al., 1995b; Ye et al., 1998) strongly support a predominantly inductive and differentiative mechanism by which VME14 and A1 cells increase the number of dopaminergic neurons from E11 cells. Their inductive and differentiative effects distinguish the VME14 and A1 cell lines from previously described mesencephalic glial cell lines that exert mainly survival-promoting activity on dopaminergic neurons (O’Malley et al., 1992, 1994; Takeshima et al., 1994; Engele et al., 1996; Panchision et al., 1998). Very recently, it has been reported that stromal cells derived from skull bone marrow produce an activity that accumulates on the cell surface and has the ability to induce TH neurons from embryonic stem (ES) cells (Kawasaki et

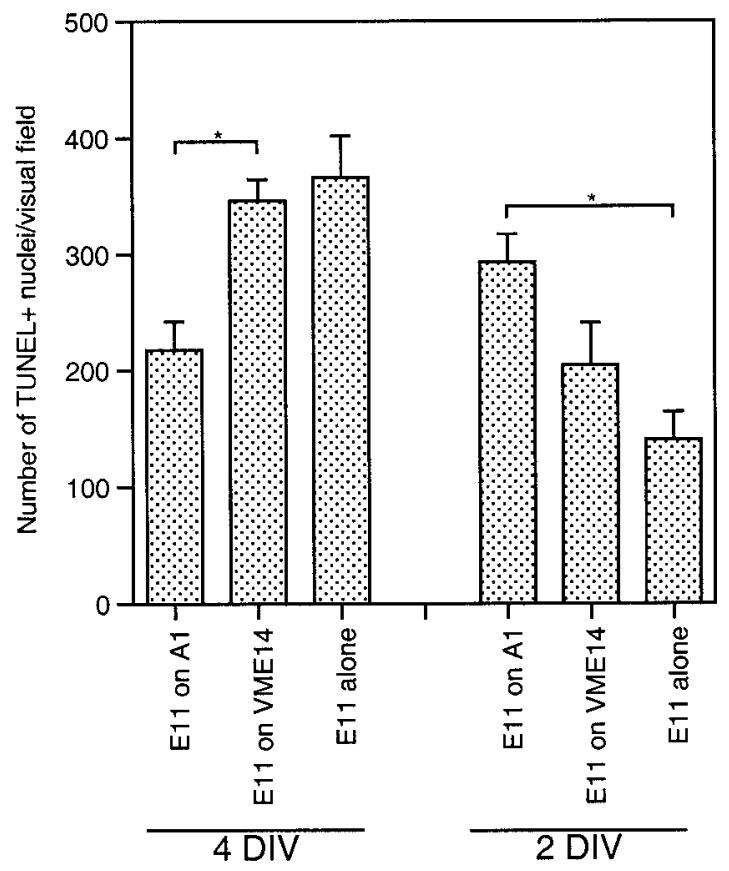

Figure 9. Histogram showing the number of TUNEL-positive $(T U N E L+)$ nuclei per visual field at $20 \times$ magnification. No significant difference in the number of TUNEL-positive nuclei was observed between E11 cells grown on a feeder layer of VME14 cells and E11 cells at any time point, indicating that the feeder layer did not enhance the survival of E11 cells. More TUNEL-positive nuclei were observed in E11-A1 cocultures after 2 DIV $\left({ }^{*} p<0.05\right)$ compared with cultures of E11 cells grown alone, indicating that enhanced survival is not the primary mechanism for the increase in TH-positive cells at this time point. At 4 DIV, fewer TUNEL-positive nuclei were observed in E11-A1 cocultures compared with the other culture conditions, suggesting that SHH-N increased cell survival at later time points.

al., 2000). It will be very interesting in the future to investigate the effects of VME14 and A1 cells in the ES cell context.

In the present experiments, medium conditioned by the differentiated VME14 or A1 cells failed to increase the number of TH-positive cells. Therefore, it is possible that cell contact between E11 mesencephalic cells and the A1 or VME14 cells is a requirement for the increased generation of TH-positive neurons. An alternative explanation is that induction of TH-positive neurons or differentiation of dopaminergic precursors is mediated by diffusible factors that must be present at high concentrations and are available only in the immediate vicinity of the feeder layer. It will be important in the future to study the molecular nature of the signals involved in the generation of dopaminergic neurons in vitro using VME14 and A1 cells. Our preliminary data suggest that additional factors other than glial cell line-derived neurotrophic factor or FGF8, which are known to have trophic and inductive effects on dopaminergic neurons (Ye et al., 1998; Granholm et al., 2000), are involved in the inductive or differentiative process, because RT-PCR failed to detect expression of either growth factor.

\section{Application of the immortalized mesencephalic cells to neural transplantation}

In experimental animal studies, fetal ventral mesencephalons at E13 or later have been used for neural transplantation. In the present study, a monolayer of the differentiated A1 cells enhanced the generation of TH-positive neurons in cultures of the E11 


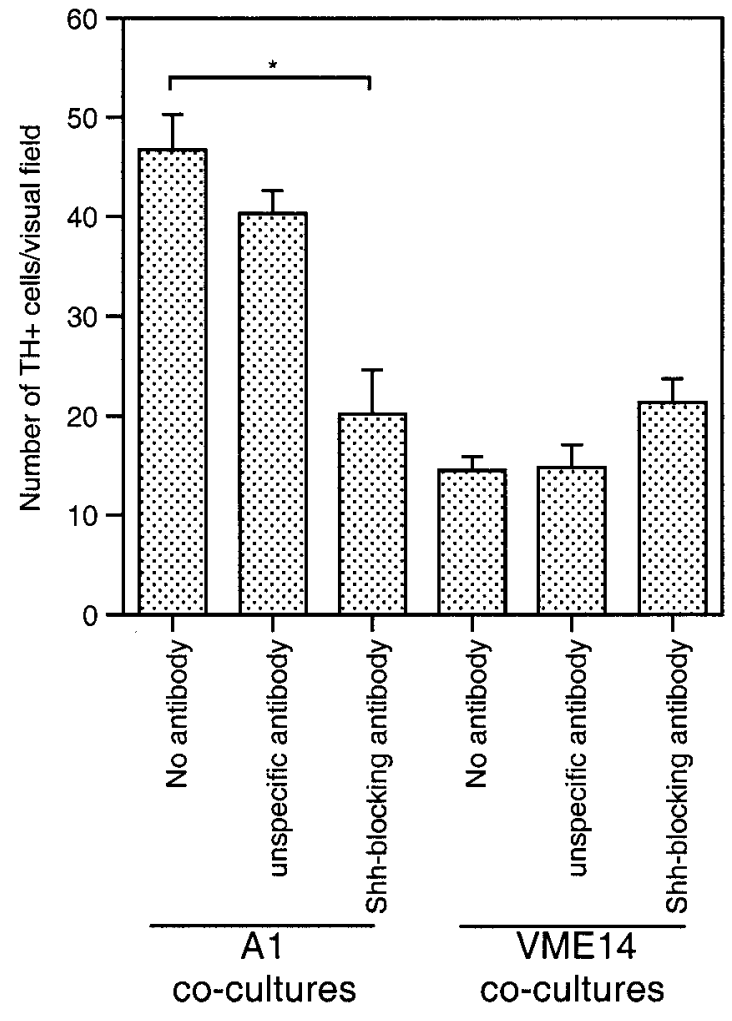

Figure 10. Histogram showing the results of SHH-N blocking experiments. Treatment of E11-A1 cocultures with $20 \mu \mathrm{g} / \mathrm{ml} \mathrm{SHH-N} \mathrm{blocking}$ antibodies decreased the number of TH-positive cells significantly $\left({ }^{*} p=\right.$ $0.005)$ and resulted in numbers comparable with E11-V ME14 cocultures. Treatment with unspecific antibody at the same concentration and treatment of E11-VME14 cocultures with blocking antibody demonstrated that this effect is not attributable to toxicity of the SHH-N blocking antibody. These results confirm that the increased efficiency of the A1 feeder layer is attributable to the additional effect of SHH-N.

mesencephalon. This procedure to enrich mesencephalic grafts with dopaminergic neurons in culture may expand the range of fetal ages useful for grafting.

Takayama et al. (1995) advocated grafting fetal mesencephalic cells together with genetically modified cells producing a neurotrophic factor, which has been shown to be a useful strategy for enhancing the effectiveness of fetal dopaminergic grafts. It has been demonstrated that immortalized neural cells grafted into the brain survive well and do not form a tumor (Snyder, 1994; Tornatore et al., 1996).

Glial cells have been shown to be a useful vehicle for neural transplantation (Yoshimoto et al., 1995; Tornatore et al., 1996). Because A1 cells differentiated into glial cells and increased the number of TH-positive cells from fetal mesencephalon, cografting of the fetal ventral mesencephalon with differentiated A1 cells may be a feasible strategy for enhancing the effects of neural transplantation in experimental models of Parkinson's disease. Studies to investigate these observations in vivo will be conducted in the future.

\section{REFERENCES}

Bjorklund A, Lindvall O (2000) Cell replacement therapies for central nervous system disorders. Nat Neurosci 3:537-544.

Dunnett SB (1991) Transplantation of embryonic dopamine neurons: what we know from rats. J Neurol 238:65-74.

Engele J, Rieck H, Choi-Lundberg D, Bohn MC (1996) Evidence for a novel neurotrophic factor for dopaminergic neurons secreted from mesencephalic glial cell lines. J Neurosci Res 43:576-586.
Ericson J, Morton S, Kawakami A, Roelink H, Jessell TM (1996) Two critical periods of Sonic Hedgehog signaling required for the specification of motor neuron identity. Cell 87:661-673.

Goslin K, Asmussen H, Banker G (1998) Rat hippocampal neurons in low-density culture. In: Culturing nerve cells, Ed 2 (Banker G, Goslin K, eds), pp 339-370. Cambridge, MA: MIT.

Granholm AC, Reyland M, Albeck D, Sanders L, Gerhardt G, Hoernig G, Shen L, Westphal H, Hoffer B (2000) Glial cell line-derived neurotrophic factor is essential for postnatal survival of midbrain dopamine neurons. J Neurosci 20:3182-3190.

Hoshimaru M, Ray J, Sah DW, Gage FH (1996) Differentiation of the immortalized adult neuronal progenitor cell line HC2S2 into neurons by regulatable suppression of the v-myc oncogene. Proc Natl Acad Sci USA 93:1518-1523.

Hynes M, Poulsen K, Tessier-Lavigne M, Rosenthal A (1995a) Control of neuronal diversity by the floor plate: contact-mediated induction of midbrain dopaminergic neurons. Cell 80:95-101.

Hynes M, Porter JA, Chiang C, Chang D, Tessier-Lavigne M, Beachy PA, Rosenthal A (1995b) Induction of midbrain dopaminergic neurons by Sonic hedgehog. Neuron 15:35-44.

Kawasaki H, Mizuseki K, Nishikawa S, Kaneko S, Kuwana Y, Nakanishi S, Nishikawa SI, Sasai Y (2000) Induction of midbrain dopaminergic neurons from ES cells by stromal cell-derived inducing activity. Neuron $28: 31-40$.

Kinto N, Iwamoto M, Enomoto-Iwamoto M, Noji S, Ohuchi H, Yoshioka H, Kataoka H, Wada Y, Yuhao G, Takahashi HE, Yoshiki S, Yamaguchi A (1997) Fibroblasts expressing Sonic hedgehog induce osteoblast differentiation and ectopic bone formation. FEBS Lett 404:319-323.

Kojima M, Hoshimaru M, Aoki T, Takahashi JB, Ohtsuka T, Asahi M, Matsuura N, Kikuchi H (1996) Expression of heat shock proteins in the developing rat retina. Neurosci Lett 205:215-217.

Kordower JH, Rosenstein JM, Collier TJ, Burke MA, Chen EY, Li JM, Martel L, Levey AE, Mufson EJ, Freeman TB, Olanow CW (1996) Functional fetal nigral grafts in a patient with Parkinson's disease: chemoanatomic, ultrastructural, and metabolic studies. J Comp Neurol 370:203-230

Miao N, Wang M, Ott JA, D'Alessandro JS, Woolf TM, Bumcrot DA, Mahanthappa NK, Pang K (1997) Sonic hedgehog promotes the survival of specific CNS neuron populations and protects these cells from toxic insult in vitro. J Neurosci 17:5891-5899.

Murphy M, Drago J, Bartlett PF (1990) Fibroblast growth factor stimulates the proliferation and differentiation of neural precursor cells in vitro. J Neurosci Res 25:463-475.

Nakafuku M, Nakamura S (1995) Establishment and characterization of a multipotential neural cell line that can conditionally generate neurons, astrocytes, and oligodendrocytes in vitro. J Neurosci Res 41:153-168

O'Malley EK, Sieber BA, Black IB, Dreyfus CF (1992) Mesencephalic type I astrocytes mediate the survival of substantia nigra dopaminergic neurons in culture. Brain Res 582:65-70.

O'Malley EK, Sieber BA, Morrison RS, Black IB, Dreyfus CF (1994) Nigral type I astrocytes release a soluble factor that increases dopaminergic neuron survival through mechanisms distinct from basic fibroblast growth factor. Brain Res 647:83-90.

Olanow CW, Kordower JH, Freeman TB (1996) Fetal nigral transplantation as a therapy for Parkinson's disease. Trends Neurosci 19:102-109.

Panchision DM, Martin-DeLeon PA, Takeshima T, Johnston JM, Shimoda K, Tsoulfas P, McKay RD, Commissiong JW (1998) An immortalized, type-1 astrocyte of mesencephalic origin source of a dopaminergic neurotrophic factor. J Mol Neurosci 11:209-221.

Poulsen KT, Armanini MP, Klein RD, Hynes MA, Phillips HS, Rosenthal A (1994) TGF beta 2 and TGF beta 3 are potent survival factors for midbrain dopaminergic neurons. Neuron 13:1245-1252.

Sladek JR, Collier TJ, Haber SN, Roth RH, Redmond DE (1986) Survival and growth of fetal catecholamine neurons transplanted into primate brain. Brain Res Bull 17:809-818.

Snyder EY (1994) Grafting immortalized neurons to the CNS. Curr Opin Neurobiol 4:742-751.

Specht LA, Pickel VM, Joh TH, Reis DJ (1981) Light-microscopic immunocytochemical localization of tyrosine hydroxylase in prenatal rat brain. I. Early ontogeny. J Comp Neurol 199:233-253.

Stockschlaeder MA, Storb R, Osborne WR, Miller AD (1991) L-Histidinol provides effective selection of retrovirus-vector-transduced keratinocytes without impairing their proliferative potential. Hum Gene Ther 2:33-39.

Studer L, Csete M, Lee SH, Kabbani N, Walikonis J, Wold B, McKay R (2000) Enhanced proliferation, survival, and dopaminergic differentiation of CNS precursors in lowered oxygen. J Neurosci 20:7377-7383.

Takahashi J, Palmer TD, Gage FH (1999) Retinoic acid and neurotrophins collaborate to regulate neurogenesis in adult-derived neural stem cell cultures. J Neurobiol 38:65-81.

Takayama H, Ray J, Raymon HK, Baird A, Hogg J, Fisher LJ, Gage FH (1995) Basic fibroblast growth factor increases dopaminergic graft sur- 
vival and function in a rat model of Parkinson's disease. Nat Med 1:53-58.

Takeshima T, Johnston JM, Commissiong JW (1994) Mesencephalic type 1 astrocytes rescue dopaminergic neurons from death induced by serum deprivation. J Neurosci 14:4769-4779.

Tornatore C, Baker-Cairns B, Yadid G, Hamilton R, Meyers K, Atwood W, Cummins A, Tanner V, Major E (1996) Expression of tyrosine hydroxylase in an immortalized human fetal astrocyte cell line; in vitro characterization and engraftment into the rodent striatum. Cell Transplant 5:145-163.

Wagner J, Akerud P, Castro DS, Holm PC, Canals JM, Snyder EY, Perlmann T, Arenas E (1999) Induction of a midbrain dopaminergic phenotype in Nurr1-overexpressing neural stem cells by type 1 astrocytes. Nat Biotechnol 17:653-659.

Wallen A, Zetterstrom RH, Solomin L, Arvidsson M, Olson L, Perlmann
T (1999) Fate of mesencephalic AHD2-expressing dopamine progenitor cells in NURR1 mutant mice. Exp Cell Res 253:737-746.

Wang MZ, Jin P, Bumcrot DA, Marigo V, McMahon AP, Wang EA, Woolf T, Pang K (1995) Induction of dopaminergic neuron phenotype in the midbrain by Sonic hedgehog protein. Nat Med 1:1184-1188.

Ye W, Shimamura K, Rubenstein JL, Hynes MA, Rosenthal A (1998) FGF and Shh signals control dopaminergic and serotonergic cell fate in the anterior neural plate. Cell 93:755-766.

Yoshimoto Y, Lin Q, Collier TJ, Frim DM, Breakefield XO, Bohn MC (1995) Astrocytes retrovirally transduced with BDNF elicit behavioral improvement in a rat model of Parkinson's disease. Brain Res 691:25-36.

Yurek DM, Sladek JR (1990) Dopamine cell replacement: Parkinson's disease. Annu Rev Neurosci 13:415-440. 